Claremont Colleges

Scholarship@ Claremont

All HMC Faculty Publications and Research

HMC Faculty Scholarship

4-1-2007

\title{
A Combinatorial Proof of Vandermonde's Determinant
}

Arthur T. Benjamin

Harvey Mudd College

Gregory P. Dresden

Washington and Lee University

\section{Recommended Citation}

Benjamin, Arthur T. and Gregory P. Dresden. "A Combinatorial Proof of Vandermonde's Determinant." The American Mathematical Monthly, Vol. 114, No. 4, pp. 338-341, April 2007.

This Article is brought to you for free and open access by the HMC Faculty Scholarship at Scholarship @ Claremont. It has been accepted for inclusion in All HMC Faculty Publications and Research by an authorized administrator of Scholarship @ Claremont. For more information, please contact scholarship@cuc.claremont.edu. 


\section{MAA}

A Combinatorial Proof of Vandermonde's Determinant Author(s): Arthur T. Benjamin and Gregory P. Dresden

Source: The American Mathematical Monthly, Vol. 114, No. 4 (Apr., 2007), pp. 338-341

Published by: Mathematical Association of America

Stable URL: http://www.jstor.org/stable/27642196

Accessed: 10/06/2013 18:56

Your use of the JSTOR archive indicates your acceptance of the Terms \& Conditions of Use, available at

http://www.jstor.org/page/info/about/policies/terms.jsp

JSTOR is a not-for-profit service that helps scholars, researchers, and students discover, use, and build upon a wide range of content in a trusted digital archive. We use information technology and tools to increase productivity and facilitate new forms of scholarship. For more information about JSTOR, please contact support@ jstor.org. 


\section{A Combinatorial Proof of Vandermonde's Determinant}

\section{Arthur T. Benjamin and Gregory P. Dresden}

We offer a combinatorial method of evaluating Vandermonde's determinant,

$$
\left|\begin{array}{ccccc}
1 & x_{0} & x_{0}^{2} & \ldots & x_{0}^{n} \\
1 & x_{1} & x_{1}^{2} & \ldots & x_{1}^{n} \\
1 & x_{2} & x_{2}^{2} & \ldots & x_{2}^{n} \\
\vdots & \vdots & \vdots & \ddots & \vdots \\
1 & x_{n} & x_{n}^{2} & \ldots & x_{n}^{n}
\end{array}\right|=\prod_{0 \leq i<j \leq n}\left(x_{j}-x_{i}\right)
$$

that is as easy as playing cards. Let $V_{n}$ denote the Vandermonde matrix with $(i, j)$ th entry $v_{i j}=x_{i}^{j}(0 \leq i, j \leq n)$. Since the determinant of $V_{n}$ is a polynomial in $x_{0}, x_{1}, \ldots, x_{n}$, it suffices to prove the identity for positive integers $x_{0}, x_{1}, \ldots, x_{n}$ with $x_{0} \leq x_{1} \leq \cdots \leq x_{n}$. We define a Vandermonde card to possess a suit and a value, where a card of Suit $i$ has a value from the set $\left\{1, \ldots, x_{i}\right\}$. (In our examples, we will let Suits $0,1,2,3$, and 4 be represented by suits $\odot, \boldsymbol{\beta}, \diamond, \varnothing$, and $\boldsymbol{\bullet}$, respectively.) Hence there are $x_{0}+x_{1}+\cdots+x_{n}$ different Vandermonde cards, but we have at our disposal an unlimited supply of each. First we do some card counting.

Card Counting Question 1. How many ways can Vandermonde cards be arranged in $n+1$ rows, where row 0 is empty, row 1 has one card of Suit 1 , row 2 has two cards of Suit 2 , row 3 has three cards of Suit $3, \ldots$, and row $n$ has $n$ cards of Suit $n$ ? The order of the cards is important, and we are allowed to repeat values of cards within each row. We call such an arrangement a Vandermonde table associated with the identity permutation $\pi=012 \ldots n$, an example of which is given in Figure 1 .

$$
\text { Col } 1 \quad \operatorname{Col} 2 \quad \operatorname{Col} 3 \quad \operatorname{Col} 4
$$

permutation $\pi$

Row 0

$$
\pi(0)=0=\odot
$$

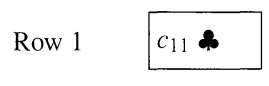

$c_{11} \in\left\{1 \ldots \ldots x_{1}\right\}$

$\pi(1)=1=\mathbf{s}$

Row $2 \quad c_{21} \diamond$

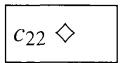

$c_{2 j} \in\left\{1 \ldots \ldots \ldots x_{2}\right\}$

$\pi(2)=2=\diamond$

Row 3
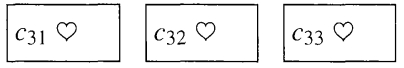

$c_{3 j} \in\{1$.

.$\left.x_{3}\right\}$

$\pi(3)=3=0$

Row 4
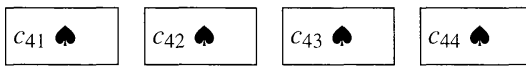

$c_{4 j} \in\{1$

..$\left.x_{4}\right\}$

$\pi(4)=4=\boldsymbol{Q}$

Figure 1. A Vandermonde table associated with the identity permutation $\pi=01234$ ( or $\pi=\odot \diamond \odot$ ). Each of the $i$ cards in row $i$ has Suit $i$ and a value from $\left\{1, \ldots, x_{i}\right\}$. Such a table can be created in $x_{1} x_{2}^{2} x_{3}^{3} x_{4}^{4}$ ways. 
Answer. For $i=0,1, \ldots, n$, the $i$ cards in row $i$ all have Suit $i$, so their values can be assigned $x_{i}^{i}$ ways. Hence, the number of arrangements is $1 x_{1} x_{2}^{2} x_{3}^{3} \cdots x_{n}^{n}$, which is the product of the diagonal entries of $V_{n}$.

Card Counting Question 2. This is the same as Question 1, but now we are given a permutation $\pi$ of the numbers 0 through $n$, say $\pi=a_{0} a_{1} \ldots a_{n}$. Here, row $i$ must contain $i$ cards from Suit $\pi(i)=a_{i}$. We call such an arrangement a Vandermonde table with permutation $\pi$. A typical table is shown in Figure 2.

Answer. Counting row by row again, there are $1 x_{\pi(1)}^{1} x_{\pi(2)}^{2} x_{\pi(3)}^{3} \cdots x_{\pi(n)}^{n}$ such tables, which is the product of the $n+1$ entries of the form $v_{\pi(i), i}$ from $V_{n}$.

Col $1 \quad \operatorname{Col} 2 \quad \operatorname{Col} 3 \quad \operatorname{Col} 4 \quad$ permutation $\pi$

Row 0

$\pi(0)=3=\varnothing$

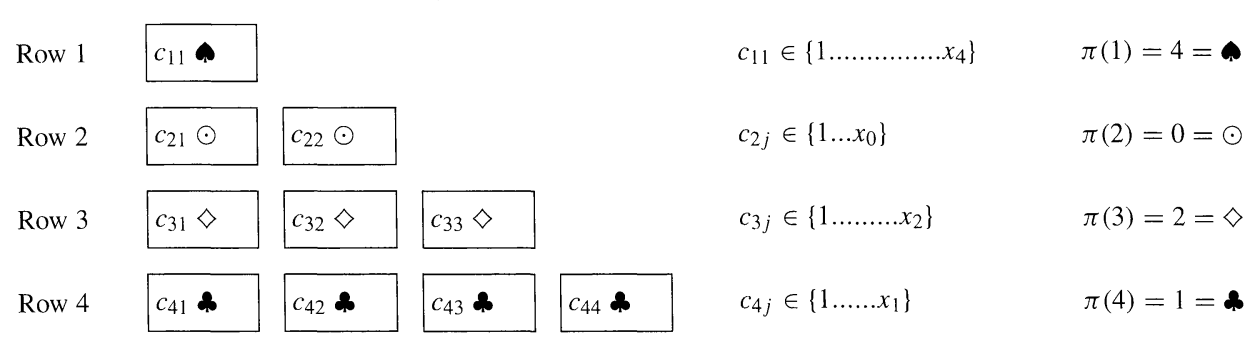

Figure 2. A Vandermonde table associated with permutation $\pi=34021$ (or $\pi=\varnothing \diamond \diamond$ ). Each of the $i$ cards in row $i$ has Suit $\pi(i)$ and a value from $\left\{1, \ldots, x_{\pi(i)}\right\}$. Such a table can be created in $x_{4} x_{0}^{2} x_{2}^{3} x_{1}^{4}$ ways.

Card Counting Question 3. Same as Question 2, but now $\pi$ is not prescribed in advance, so $\pi$ can be any permutation of $\{0, \ldots, n\}$. As before, each row is assigned a different suit and row $i$ contains $i$ cards of the assigned suit. For this unrestricted problem, such an arrangement is simply called a Vandermonde table.

Answer. Sum the answer to Question 2 over all possible permutations of $0, \ldots, n$. In other words, the number of ways to create a Vandermonde table is the permanent of $V_{n}$.

Card Counting Question 4. Question 3 again, but now we count those arrangements with even permutations positively and those arrangements with odd permutations negatively.

Answer. By definition, this is the determinant of $V_{n}$.

It remains to show that the answer to Question 4 also equals $\prod_{0 \leq i<j \leq n}\left(x_{j}-x_{i}\right)$. For a given Vandermonde table $C$ let the cards of row $i$ be denoted by $C_{i 1}, C_{i 2}, \ldots, C_{i i}$, with values $c_{i 1}, c_{i 2}, \ldots, c_{i i}$. We say that card $C_{i j}$ is small if $c_{i j} \leq x_{j-1}$. For example, any card in column 1 with a value less than or equal to $x_{0}$ (such as any card of Suit 0 ) is small.

Card Counting Question 5. How many Vandermonde tables have no small cards?

Answer. Let $C$ be a Vandermonde table with no small cards. Since column 1 must not contain any cards of Suit 0 , Suit 0 must be assigned to the empty row 0 . Next, since column 2 must not contain any cards with value less than or equal to $x_{1}$ (such as any 
card of suit 1), we must assign suit 1 to row 1 . Continuing this reasoning, row 2 must have Suit $2, \ldots$, and row $n$ must have Suit $n$. Thus $C$ must be associated with the identity permutation. Furthermore, to avoid small cards in the first column, the values of the cards $C_{11}, \ldots, C_{n 1}$ can be assigned in $\left(x_{1}-x_{0}\right)\left(x_{2}-x_{0}\right)\left(x_{3}-x_{0}\right) \cdots\left(x_{n}-x_{0}\right)$ ways. Likewise, the values of the cards in the second column can be assigned in $\left(x_{2}-\right.$ $\left.x_{1}\right)\left(x_{3}-x_{1}\right) \cdots\left(x_{n}-x_{1}\right)$ ways, and so on down to the single card of Suit $n$ in the last column, with a value that can be assigned in $x_{n}-x_{n-1}$ ways. We conclude that there are $\prod_{0 \leq i<j \leq n}\left(x_{j}-x_{i}\right)$ Vandermonde tables with no small cards.

We say that a Vandermonde table is good if it has no small cards and is bad if it has at least one small card. Note that since the identity permutation is even, all of the good tables are counted positively in the determinant of $V_{n}$.

To complete the proof of Vandermonde's expansion, it suffices to show that every bad Vandermonde table can be paired up with another bad Vandermonde table with a permutation of opposite parity. Thus, when the determinant of $V_{n}$ sums over all Vandermonde tables, the bad tables cancel each other out. When the dust settles, only the good tables (all counted positively) remain standing.

Now let $C$ be a bad Vandermonde table with permutation $\pi=a_{0} a_{1} \ldots a_{n}$. We define the first small card of $C$ to be the small card $c_{i j}$ where $j$ is as small as possible, and if column $j$ has more than one small card, then we choose $i$ to be as large as possible. In other words, we look for small cards from bottom to top, beginning in column 1 .

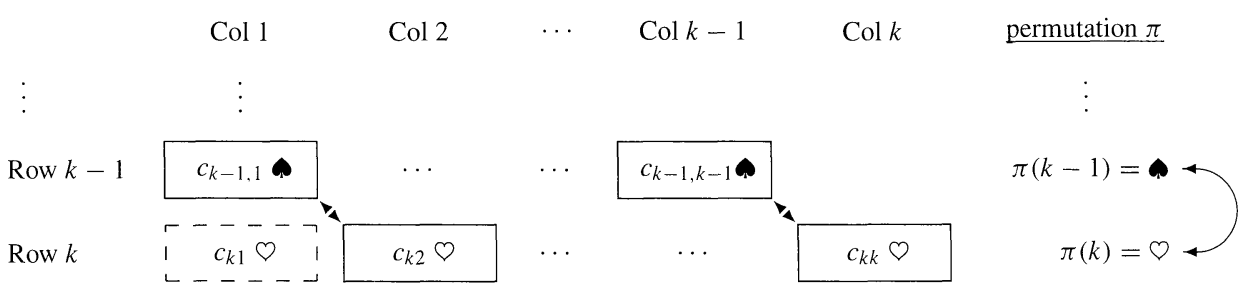

Figure 3. When the first small card occurs in the first column at card $C_{k 1}$, simply swap the cards of row $k-1$ with the cards $C_{k 2}, \ldots, C_{k k}$, and change the suit of card $C_{k 1}$.

Suppose that the first small card of $C$ occurs in column 1, say card $C_{k 1}$ for some $1 \leq k \leq n$. Then, since $C_{k 1}$ is small, $c_{k 1} \leq x_{0}$, and since it is the first small card, there are no small cards below it; that is, when $i>k, c_{i 1}>x_{0}$. For definiteness, suppose that the cards in row $k-1$ have Suit $\pi(k-1)=\boldsymbol{a}$ and that the cards in row $k$ have Suit $\pi(k)=\varnothing$. (We make no assumptions about the suit number for hearts or spades.) Now consider the Vandermonde table $C^{\prime}$ obtained by swapping all $k-1$ spade cards with all of the heart cards except for card $C_{k 1}$. Then change the suit of card $C_{k 1}$ from hearts to spades. The suit change from hearts to spaces is legal because $c_{k 1} \leq x_{0}$, which is a legal value for all suits. (Here we are exploiting the fact that $x_{0} \leq x_{1} \leq \cdots \leq x_{n}$.) Notice that $C_{k 1}$ is still the first small card of $C^{\prime}$, albeit with a new suit, and thus if we apply the swapping procedure to $C^{\prime}$, we obtain $C$. That is, $\left(C^{\prime}\right)^{\prime}=C$. Furthermore, $C^{\prime}$ has permutation $\pi^{\prime}=a_{0} a_{1} \ldots a_{k} a_{k-1} \ldots a_{n}$. Permutations $\pi$ and $\pi^{\prime}$ have opposite parity since they differ by the transposition of hearts and spades (see Figure 3).

Now suppose that the first small card of $C$ occurs in column $j$ with $j \geq 2$, say at card $C_{k j}$. Then $c_{k j} \leq x_{j-1}$, and there are no small cards anywhere in columns 1 through $j-1$ nor below card $C_{k j}$ in column $j$. As before, suppose that the cards of row $k$ have the heart suit and that the cards of row $k-1$ have the spade suit. Create a new Vandermonde table $C^{\prime}$ by swapping the first $j-1$ cards of rows $k-1$ and $k$, 

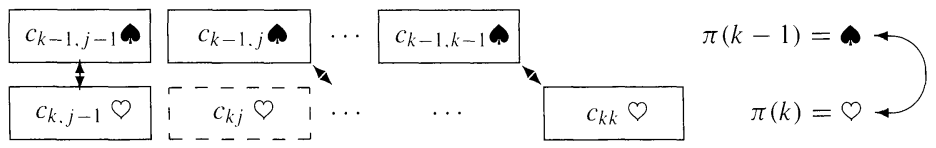

Figure 4. When $C_{k j}$ is the first small card and $j \geq 2$, then swap the first $j-1$ cards of row $k-1$ with the first $j-1$ cards of row $k$, change the suit of card $C_{k j}$, then swap the remaining cards of rows $k-1$ and $k$. In the new Vandermonde table, card $C_{k j}$ remains the first small card.

leaving card $C_{k j}$ in its place, but changing its suit from hearts to spades, then swapping the remaining $k-j$ cards of rows $k-1$ and $k$, as in Figure 4 .

Why is it legal to change the suit of card $C_{k j}$ from hearts to spades? Since $C_{k j}$ was the first small card, then the spade card $C_{k-1, j-1}$ is not small and therefore has a value strictly greater than $x_{j-2}$. Thus all spade cards can take on values less than or equal to $x_{j-1}$. Since $C_{k j}$ is small, its value is at most $x_{j-1}$, so changing it from hearts to spades is allowable.

As before, $C_{k j}$ remains the first small card of $C^{\prime}$, so $\left(C^{\prime}\right)^{\prime}=C$ and $C^{\prime}$ has permutation $\pi^{\prime}$, which has opposite parity of $\pi$ since they differ by a transposition. Thus there is a one-to-one correspondence between the positively counted Vandermonde tables with small cards and the negatively counted Vandermonde tables with small cards. Therefore the determinant of $V_{n}$ is the number of Vandermonde tables with no small cards, namely, $\prod_{0 \leq i<j \leq n}\left(x_{j}-x_{i}\right)$, as desired.

Remark. For another combinatorial proof of Vandermonde's determinant, where the cancellation occurs in the product instead of the sums, see the short paper by Ira Gessel [1].

\section{REFERENCES}

1. I. Gessel, Tournaments and Vandermonde's Determinant, J. Graph Theory 3 (1979) 305-307.

Harvey Mudd College, Claremont, CA 91711

benjamin@math.hmc.edu

Washington and Lee University, Lexington, VA 24450

dresdeng@wlu.edu

\section{Evaluation of Some Improper Integrals Involving Hyperbolic Functions}

\section{Michael A. Allen}

In this note I present a result that seems elementary enough to be added to the list of tricks for evaluating integrals taught in a complex variables course, but one to which I have been unable to find any reference. It gives a straightforward procedure that can be used to evaluate a class of integrals some of which do not appear in [1] and for which Mathematica 5.1 [2] generates expressions involving exotic special functions that it cannot simplify further. 